\title{
Editorial
}

Int Neurourol J 2010;14:201-202

doi: 10.5213/inj.2010.14.4.201

pISSN 2093-4777 · eISSN 2093-6931

\section{Ischemic Tolerance Induced by Cerebral Preconditioning: New Urologic Research Area}

\author{
Khae Hawn Kim \\ Associate Editor \\ Department of Urology, Gachon University Gil Hospital, Gachon University of Medicine and Science, Incheon, Korea \\ E-mail: kimcho99@gilhospital.com
}

Voiding function and dysfunction, bladder physiology and pharmacology covering peripheral and central nerve system (CNS) have been given much attention during the last decades as confirmed by countless researches and clinical articles. In contrast, cerebral preconditioning and associated brain lesions in urologic field generated only a few clinical articles regarding to ischemic stroke [1,2]. This difference is most probably due to the commercial success of detrusor overactivity treatment with antimuscarinics, which currently is a several billion dollar market worldwide, and indifference to the development of new drugs, which directly affect on the CNS.

In this issue of the Journal, Bhuiyan and Kim [3] illustrated the likelihood of an overview with ishemic brain lesion associated with voiding dysfunction and possibility of applying cerebral preconditioning to urologic field. In ischemically damaged brain, detrusor overactivity seems to be from a deactivation of prefrontal cortex, but detrusor dysfunction is affected by various parts of brain. Localization of the ischemic lesion is not associated with any specific type of lower urinary tract dysfunction. Furthermore, it is suggested that the changes of neurotransmitters release after ichemic brain damage may affect the voiding dysfunction. Now, in Urology, first-line treatments for voiding dysfunction include lifestyle modification, behavioral therapy and pelvic floor muscle training. Muscarinic receptor antagonists are the first-line drug therapy. However, they are related to the typical anticholinergic side effects of dry mouth, somno- lence, constipation and blurred vision, therefore compliance with therapy becomes often poor, and the improvement of quality of life fails. In addition, this issue introduces several neurotransmitters release during ischemic stroke associated with neuronal membrane receptors; excitatory amino acids such as the a-amino-3-hydroxy-5-methyl-4-isoxazole-propionic acid receptors and major inhibitory neurotransmitter system such as pharmacological stimulation of the $\gamma$-aminobutyric acid-ergic system. However, agents with this profile and even with these mechanisms applied are not under development currently much more. If the further studies focused on preconditioning strategy and related interventions, such as remote preconditioning and pharmacological preconditioning, revealed the way urologist want them to, they would make a valuable contribution to the treatment of voiding dysfunction for which there is an urgent and the future demands for effective therapy. The efforts of academic and drug industry researchers should be directed toward developing agents with therapeutic effects on CNS and associated preconditioning, as it would be not only scientifically also clinically rewarding.

\section{REFERENCES}

1. Han KS, Heo SH, Lee SJ, Jeon SH, Yoo KH. Comparison of urodynamics between ischemic and hemorrhagic stroke patients: can we suggest the category of urinary dysfunction in patients with cere- 


\section{Kim • Ischemic Tolerance Induced by Cerebral Preconditioning: New Urologic Research Area}

brovascular accident according to type of stroke? Neurourol Urodyn 2010;29:387-90.

2. Griffiths D, Tadic SD. Bladder control, urgency, and urge incontinence: evidence from functional brain imaging. Neurourol Urodyn
2008;27:466-74.

3. Bhuiyan MI, Kim YJ. Mechanisms and prospects of ischemic tolerance induced by cerebral preconditioning. Int Neurourol J 2010; 14:203-212. 